\title{
Macrophage inflammatory protein-3 $\alpha$ influences growth of K562 leukemia cells in co-culture with anticancer drug-pretreated HS-5 stromal cells
}

\author{
Ying Chu Lee ${ }^{\mathrm{a}}$, Tzeon-Jye Chiou ${ }^{\mathrm{b}, \mathrm{c}}$, Woan-Fang Tzeng ${ }^{\mathrm{d}}$, Sin Tak Chu ${ }^{\mathrm{a}, \mathrm{e}, *}$ \\ a Institute of Biological Chemistry, Academia Sinica, Taipei, Taiwan \\ ${ }^{\mathrm{b}}$ Division of Transfusion Medicine, Department of Medicine, Taipei Veterans General Hospital, Taiwan \\ ' School of Medicine, National Yang-Ming University, Taipei, Taiwan \\ d Department of Life Science, Fu-Jen Catholic University, Taipei, Taiwan \\ e Institute of Biochemical Sciences, College of Life Science, National Taiwan University, Taipei, Taiwan
}

\section{A R T I C L E I N F O}

\section{Article history:}

Received 28 March 2008

Received in revised form 15 April 2008

Accepted 15 April 2008

Available online 30 April 2008

\section{Keywords:}

Bone marrow stromal cell

Cytokines

Leukemic cell

\begin{abstract}
A B S T R A C T
Stromal cell monolayers have been an important means of studying the regulation of hematopoiesis, because they produce cytokines. Cytosine arabinoside, vincristine, daunorubicin, and doxorubicin are common drugs for hematological cancer therapy, and they may have some effects on bone marrow stroma during chemotherapy. The aim of this study was to elucidate interactions between the bone marrow stromal microenvironment and leukemic cells after drug treatment. We tested the hypothesis that human HS-5 stromal cells, pretreated with anticancer drugs, affected the growth of leukemic K562 cells by changing the cytokines in the culture microenvironment. Thereafter, proliferation of K562 cells increased nearly 2.5-fold compared the co-cultivation with drugs-pretreated HS-5 stromal cells and drugs-untreated HS-5 stromal cells. The results indicated that co-cultivation with HS-5 stromal cells pretreated with drugs caused significant $\mathrm{K} 562$ cell proliferation. Cytokines in the microenvironment were detected via the RayBio ${ }^{\circledR}$ Human Cytokine Antibody Array Membrane. The levels of the cytokines CK $\beta$, IL-12, IL-13, IGFBP-2, MCP-1, MCP-3, MCP-4, MDC, MIP-1 $\beta$ and MIP- $1 \delta$ were decreased, with a particularly marked decrease in MIP-3 $\alpha$. In co-culture medium, there was a 20 -fold decrease in MIP-3 $\alpha$ in daunorubicin-pretreated HS-5 cells and at least a 3-fold decrease in Ara-C-pretreated cells. This indicated a significant effect of anticancer drugs on the stromal cell line. Using phosphorylated Erk and pRb proteins as cell proliferation markers, we found that phosphorylation of these markers in K562 cells was inhibited during co-cultivation with drug-pretreated stromal cells in MIP-3 $\alpha$-supplemented medium and restored by MIP-3 $\alpha$ antibody supplement. In conclusion, anticancer drug pretreatment suppresses the negative control exerted by HS- 5 cells on leukemic cell proliferation, via modulation of cytokines in the microenvironment, especially at the level of MIP-3 $\alpha$.
\end{abstract}

(c) 2008 Elsevier Ireland Ltd. All rights reserved.

\section{Introduction}

The growth and differentiation of most types of hematopoietic cells in vivo require direct contact with stem cells. The bone marrow provides the basic microenvironment for hematopoiesis, and stromal cells are the principal components of this microenvironment. In vitro studies have shown that stromal cells regulate hematopoiesis by secreting cytokines that are important for normal proliferation and differentiation. Recently, some data have suggested that bone marrow stromal cells are involved in the trafficking of malignant $\mathrm{B}$ cells within the bone marrow microenvironment by cell con-

\footnotetext{
* Corresponding author at: Institute of Biological Chemistry, Academia Sinica, P.O. Box 23-106, Taipei, Taiwan. Tel.: +886 2 23620261x4121; fax: +8862 33664073.

E-mail address: stc316@gate.sinica.edu.tw (S.T. Chu).
}

tact or production of cytokines. Thus, intercellular cross-talk with activating and suppressive cytokines reflects a common mechanism in the regulation of cell growth in the system (Jiang et al., 1998; Badillo and Flake, 2006; Wu et al., 2005; Torok-Storb et al., 1999). In treatment with cytostatic drugs, toxicity for hematopoietic progenitor cells is a dose-limiting factor. This may increase the incidence of chemotherapeutic failure in leukemia patients. Stromal cell-based assays have been used to study the growth requirements of hematopoietic cells, since leukemic cells originate from their normal counterparts and also reside within the bone marrow microenvironment, and it is, therefore, likely that stromal cells influence the growth of leukemic cells. Basically, cytostatic drugs damage normal and tumor cells, and therefore, change the bone marrow microenvironment. Cordero et al. (2004) showed that development of the hematopoietic system is influenced by the intrinsic qualities of hematopoietic stem cells and their supportive 
stromal cells. This report also suggests that soluble substance(s) produced from stromal cells play a role in the growth of leukemic cells (Frikin et al., 1993; Aoyagi et al., 1996). In leukemia patients, anticancer drugs may affect bone marrow stromal cells by altering stromal cell secretory cytokines, which influence the leukemic cells. A previous study has shown that stromal elements are not transplantable; the capacity of the recipient's stroma to support hematopoiesis following chemotherapy and bone marrow transplantation (BMT) is important (Simmons et al., 1987). It implies that the microenvironment of bone marrow is one of the critical factors for success in chemotherapy and BMT.

The HS-5 cell line is a transformed cell line HPV-16 E6/E7 derived from human bone marrow stromal cells, and it supports growth of hematopoietic progenitor cells (Roecklein and TorokStorb, 1995). HS-5 has been reported to improve AML cell survival in vitro and attenuate chemotherapy-induced cell killing (Garrido et al., 2001). Observation of stromal cells pretreated with anticancer drugs shows that they sustain cell growth in vitro better than in vivo. We tested the influence of four anticancer drugs on cell growth by co-cultivation of the AML cell line (K562) with HS-5 stromal cells. Based on previous data, we hoped to clarify the influence of anticancer drugs on stromal cells, an influence which should be significant. The purpose of our study was to investigate the effect of cytosine arabinoside (Ara-C), daunorubicin (Dau), doxorubicin (Dox) and vincristine (Vin) on stromal cells (HS-5). The experiment was carried out in vitro by co-cultivation of drug-pretreated HS-5 cells with K562 cells, to assess the cell growth support of stromal cells.

\section{Material and methods}

2.1. Cells

The human myeloid leukemic K562 cell line which corresponds to AML cell line, derived from a chronic myeloid leukemia patient, was kindly provided by Dr. L.C. Yu of the Institute of Biochemical Sciences of National Taiwan University. The cell line was routinely cultured in RPMI 1640 medium supplemented with $10 \%$ newborn calf serum (NCS). The human stromal cell line HS-5 was a gift from Dr. Y.H. Huang at Taipei Medical Uni- versity. The HS-5 cell line was maintained in RPMI 1640 containing 10\% NCS and routinely used for the support of K562 leukemic cell differentiation.

\subsection{Chemicals}

Ara-C was purchased from David Bull Laboratories (DBL, USA). A stock solution of $100 \mathrm{mg} / \mathrm{ml}$ was made and stored at $-20^{\circ} \mathrm{C}$. Daunorubicin, doxorubicin, vincristine, and MTT [3-(4,5-dimethythiazolyl)-2,5-diphenyl-2H-tetrazolium bromide] were purchased from Sigma (MO, USA). All of the other chemicals were reagent grade. The Human MIP-3alpha ELISA Kit was obtained from RayBiotech. Phosphop44/42 MAP kinase antibody (for Thr202/Tyr204, p-Erk) was obtained from Cell Signaling Technology (\#9101). Phosphorylated Rb antibody (for phospho-S249) was obtained from abcam (ab4788). Anti-Erk 1 antibody was purchased from Santa Cruz Biotechnology (sc-93). Anti-human CCL20/MIP-3 $\alpha$ antibody was purchased from R\&D Systems (ab9349).

\subsection{MTT assay for cell viability}

MTT can be reduced to a blue formazan dye and thereby assesses cell viability by measuring mitochondrial function. HS-5 stromal cells were seeded into six-well plates and cells were incubated with various amounts of anticancer drugs. After incubation, cells were washed three times with PBS buffer containing $0.2 \%$ NCS and then incubated with $2.5 \mathrm{mg} / \mathrm{ml}$ MTT for $40 \mathrm{~min}$ at $37^{\circ} \mathrm{C}$. DMSO $(0.5 \mathrm{ml})$ was then added to dissolve the resultant crystals for $30 \mathrm{~min}$ at $37^{\circ} \mathrm{C}$. OD 570 was measured, and a reduction indicated loss of cell viability and growth.

\subsection{Non-contact co-culture}

Transwell clear plates (Corning, NY, USA) with $0.4 \mu \mathrm{m}$ pore size polyester membrane were used for non-contact co-culture. Co-cultures were initiated by seeding K562 cells into the transwell insert on a monolayer of HS- 5 cells $\left(1 \times 10^{5}\right.$ cells $)$ grown in six-well plates that contained 5\% NCS in RPMI 1640 medium. All experiments used K562 cells at a density of $1 \times 10^{4}$ cells $/ 4 \mathrm{ml}$ on the first day, and then changed half of the medium, which was finally made up to $5 \mathrm{ml}$ with fresh medium on day 4 . K562 cells were harvested after a total of 7 days by pipetting the medium that contained the cells to rule out the presence of stromal cells, and then centrifuging at $200 \times \mathrm{g}$ (KUBOTA 1920, Japan) for $5 \mathrm{~min}$. Cell counts were taken and the cell-free medium was retained for the cytokine assay. Before co-culturing with K562 cells, HS- 5 cells were incubated in the presence or absence of drugs for $48 \mathrm{~h}$. Following removal of the drugs, fresh medium was added to the HS- 5 cells for $24 \mathrm{~h}$, then the medium was removed and co-cultured with K562 cells in RPMI 1640 medium that contained 5\% NCS.
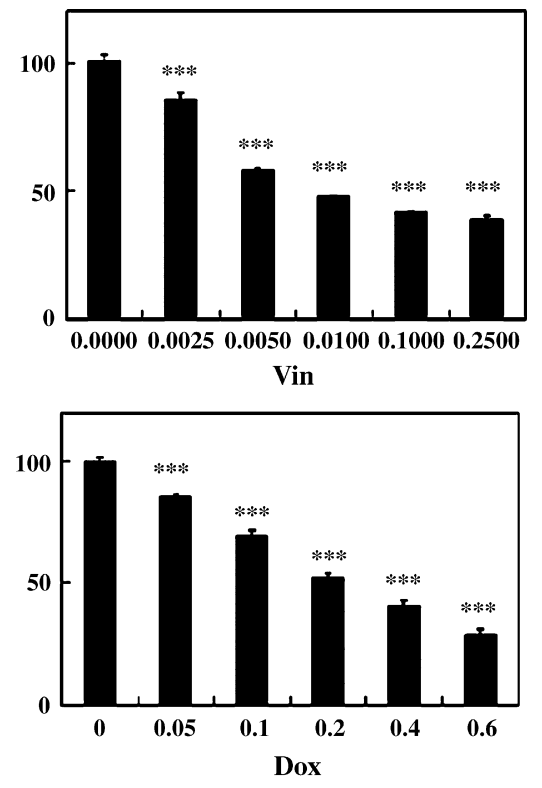

Concentration, $\mu \mathrm{M}$

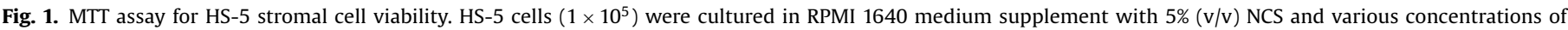

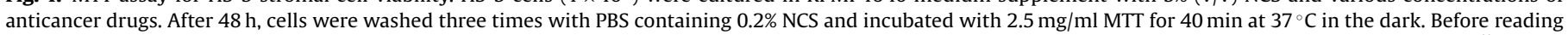

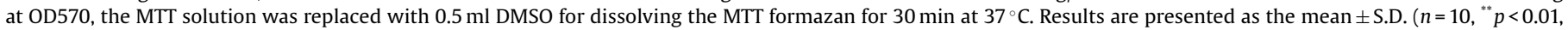
${ }^{* * *} p<0.001$ ). Significantly different from corresponding control group is shown at $p$-value. 


\subsection{Cytokines assays in stromal conditioned medium (CM)}

The levels of cytokines in CM were measured by a RayBio Human Cytokine Antibody Array V \& 5.1 Map. This assay employs a qualitative Western screening technique. The standard array matrix consisted of an $11 \times 8$ dot grid on a $20 \mathrm{~mm} \times 30 \mathrm{~mm}$ nitrocellulose membrane with 79 unique capture antibodies. Array kit included an instruction manual and with the biotinylated-antibodies solution and chemiluminescent substrate. The cytokine array membrane was incubated with $1 \mathrm{ml}$ 7-day-co-culture medium or 7-day control medium for $2 \mathrm{~h}$, and then the membrane was washed three times with washing buffer I for 5 min each, and then washing buffer II for $5 \mathrm{~min}$ each. Cytokine was detected using cytokine antibody for $1 \mathrm{~h}$, and then followed by HRP-labeled strepavidin incubation for $1 \mathrm{~h}$. The dilution used for each of these reagents was suggested by the instructions contained in the assay kit. Reactive spots were visualized by enhanced chemiluminescence (ECL) (Amersham Pharmacia Biotech UK Limited) with exposure to X-ray film. Estimation of change in these cytokines was based on the relative ratio to the control, using a non-linear dynamic analysis method with Phoretix ${ }^{\mathrm{TM}}$ 2D software.

\subsection{ELISA}

Cell culture medium was harvested after 7 days and measured by ELISA according to the manufacturer's instructions (Ray Biotech, USA).

\subsection{Western blot analysis}

The dissolved proteins in the sample buffer were resolved by SDS-PAGE on a $12 \%$ acrylamide gel slab. Proteins were transferred onto a PVDF membrane by electrotransfer $(17 \mathrm{~V})$ at room temperature for $18 \mathrm{~h}$. The transferred proteins were detected using HRP-conjugated antibody diluted to $1: 1000,1: 3000$ and 1:10000 for p-Erk, $\mathrm{p}-\mathrm{Rb}$ and Erk-1, respectively. The reactive bands were visualized using an ECL kit and exposed on X-ray film.

\subsection{Statistical analysis}

The statistical analysis was conducted using one-way ANOVA with Dunnett's post-test using GraphPad InStat version 3.00 for Windows (GraphPad Software, San Diego, CA, USA).

\section{Results}

\subsection{Evaluation of cytotoxic effect of Ara-C on HS-5 cells}

Before co-cultivation of K562 cells with drug-pretreated HS-5 cells, we investigated the effect of these drugs on HS-5 stromal cell viability, using the MTT assay. Based on the chemotherapy information, we used a test concentration lower than the level in human blood serum (Seymour et al., 1996; Holstein and Hohl, 2001). HS-5 cells were treated with various concentrations of each drug for $48 \mathrm{~h}$. The data showed that cell viability was inversely proportional to anticancer drug concentration (Fig. 1). When the concentration of drugs reached a certain level, the viability of HS- 5 cells was reduced by $50 \%$, which suggested the cytotoxicity of these anticancer drugs on HS- 5 cells. The $\mathrm{LD}_{50}$ dose of $\mathrm{AraC}$, vincristine, daunorubicin and doxorubicin on HS- 5 cells was $1.0,0.01,0.1$ and $0.2 \mu \mathrm{M}$, respectively. Under such circumstances, we suppose that the microenvironment will be changed in half of the HS- 5 cells, and secretion of cytokines will be stopped. Based on these data, we used the $\mathrm{LD}_{50}$ concentration to pretreat HS-5 cells before co-culturing with K562 cells. We aimed to establish whether the microenvironment of drugdamaged HS-5 cells had an effect on K562 cell growth.

\subsection{Effects of microenvironment on cell proliferation}

K562 cells undergo differentiation induced by various agents and their optimal microenvironment, including co-culture with stromal cells (Torok-Storb et al., 1999; Barak and Ben-Ishay, 1994). After undergoing cell differentiation, proliferation should be slowed down. Suppression of leukemic cell proliferation by stromal cells is well known. Since K562 cell differentiation is induced by co-culturing with HS-5 cells, we examined the effect of the microenvironment on cell growth. Fig. 2A shows the number of
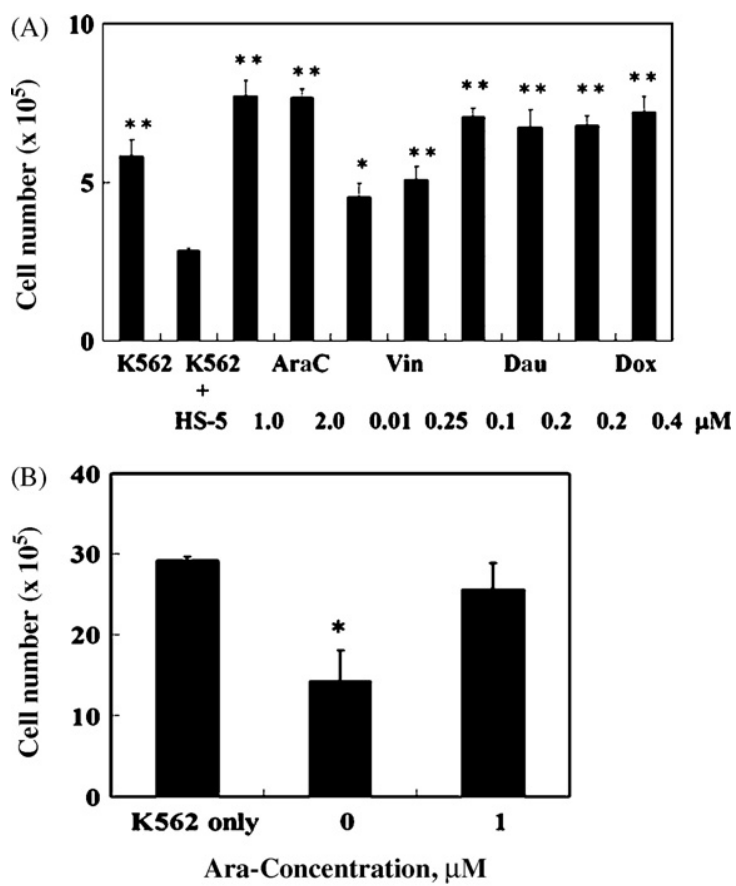

(C)
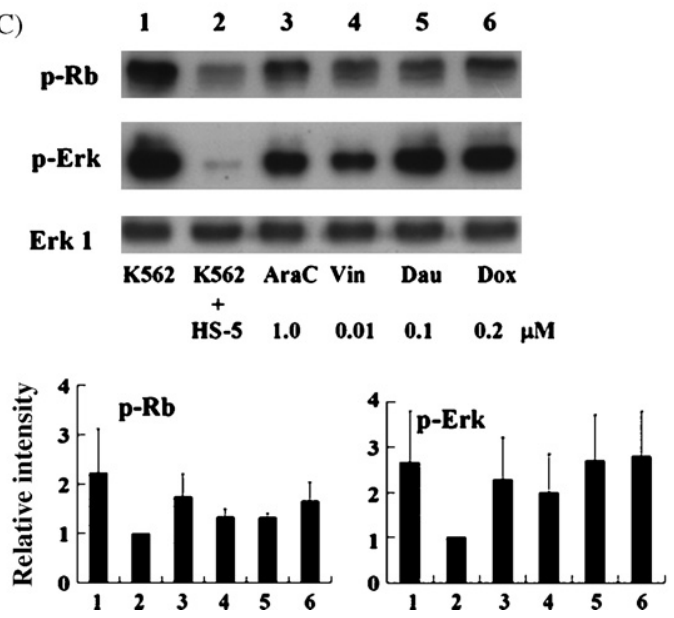

Fig. 2. Effect of co-cultured HS-5 cells on K562 cells proliferation. (A) K562 cells $\left(1 \times 10^{4}\right.$ cells) were co-cultured with anticancer-drug-pretreated HS- 5 cells in RPMI1640 medium for 7 days. The cell number of K562 counted via hemocytometer indicates the $\mathrm{K} 562$ cell proliferation. The data shown represent three independent experiments. ${ }^{*} p<0.01,{ }^{* *} p<0.001$. (B) K562 cells $\left(1 \times 10^{4}\right.$ cells $)$ were cultured alone or with $5 \mathrm{ml} \mathrm{CM}$ for 14 days. The conditional media were collected from Ara-C untreated HS- 5 cells or $1.0 \mathrm{mM}$ Ara-C pretreated HS- 5 cells. The data shown represent three independent experiments. ${ }^{*} p<0.05$. (C) Effect of drug-pretreated HS- 5 cells on phosphorylation of $\mathrm{Rb}$ and Erk protein on K562 cells. K562 cells were co-cultured with drugs pretreated HS-5 cells (lane 3-6) or cultured alone (lane 1) for 7 days. Levels of phospho-Rb and phospho-Erk were estimated by Western blot analysis using specific antibodies. K562 cells co-cultured with untreated HS-5 cells for 7 days were used as control (lane 2). The upper panel in (C) represents blots of $\mathrm{p}-\mathrm{Rb}$ and $\mathrm{p}$ Erk activation. Lower panel in (C) represents densities of p-Rb and p-Erk. Data are mean \pm S.D. of three independent experiments. Using anti-Erk antibody for recognizing Erk was an internal control. Significantly different from corresponding control group is shown at $p$-value.

K562 cells cultured alone or co-cultured with drug-pretreated HS-5 cells. Cultured K562 cells alone would keep proliferation constantly. Co-cultivation of K562 cells with untreated HS-5 cells showed fewer cells than for K562 cells cultured alone or co-cultured with drugpretreated HS-5 cells. In comparison with K562 cell proliferation in RPMI 1640 medium alone (Fig. 2A), co-culture with drug-pretreated HS-5 cells for 7 days maintained K562 cell proliferation. These data 


\begin{tabular}{|c|c|c|c|c|c|c|c|c|c|c|c|}
\hline & A & B & c & D & E & $\mathbf{F}$ & G & H & $\mathbf{I}$ & J & $\mathbf{K}$ \\
\hline 1 & Pos & Pos & Pos & Pos & Neg & Neg & ENA-78 & GCSF & GM-CSF & GRO & GRO- $a$ \\
\hline 2 & $1-309$ & $\mathrm{IL}-1 \alpha$ & IL-1 $\beta$ & IL-2 & IL-3 & IL-4 & IL-5 & IL- 6 & IL-7 & IL-8 & IL-10 \\
\hline 3 & IL-12 p40p70 & IL-13 & IL-15 & IFN- $y$ & MCP-1 & MCP-2 & MCP-3 & MCSF & MDC & MIG & MIP-1 $\beta$ \\
\hline 4 & MIP-18 & RANTES & SCF & SDF-1 & TARC & TGF- $\beta 1$ & TNF- $\alpha$ & TNF- $\beta$ & EGF & IGF-I & Angiogenin \\
\hline 5 & Oncostatin M & Thrombopoietin & VEGF & PDGF-BB & Leptin & BDNF & BLC & $C k \beta$ 8-1 & Eotaxin & Eotaxin-2 & Eotaxin-3 \\
\hline 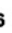 & $\mathrm{FGF}-4$ & FGF-6 & FGF-7 & FGF-9 & Flt-3 Ligand & Fractalkine & GCP-2 & GDNF & HGF & IGFBP-1 & IGFBP-2 \\
\hline & IGFBP-3 & IGFBP-4 & IL-16 & IP-10 & LIF & LIGHT & MCP-4 & MIF & MIP-3 $\alpha$ & NAP-2 & NT-3 \\
\hline & NT-4 & Osteoprotegerin & PARC & PIGF & TGF- $\beta 2$ & TGF- $\beta 3$ & TIMP-1 & TIMP-2 & $\mathrm{Neg}$ & Pos & Pos \\
\hline
\end{tabular}

\section{RayBio® Human Cytokine Antibody Array 5.1 (Cat\# H0109805)} RayBiotech, Inc.

(B)

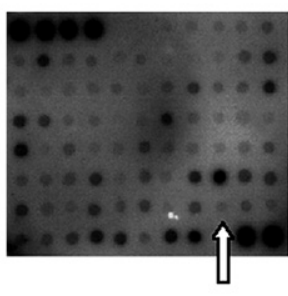

Medium only

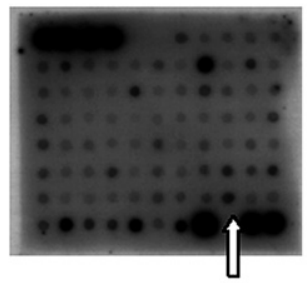

$\mathrm{K} 562+\mathrm{HS}-5$

Ara C $1.0 \mu \mathrm{M}$

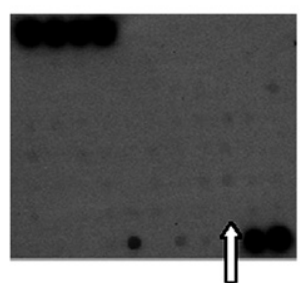

K562 only

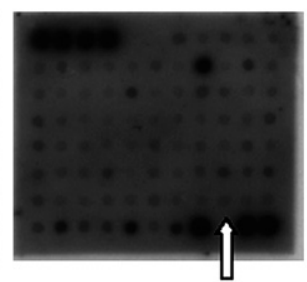

$\mathrm{K} 562+\mathrm{HS}-5$

Vin $0.01 \mu \mathrm{M}$

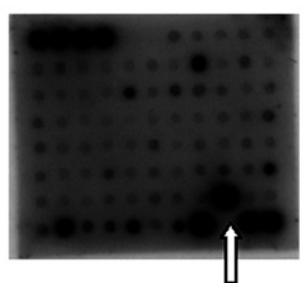

HS-5 only

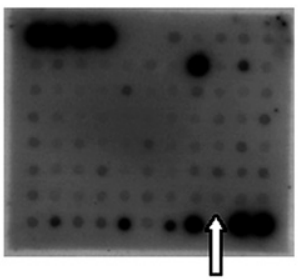

$\mathrm{K} 562+\mathrm{HS}-5$

Dau $0.1 \mu \mathrm{M}$

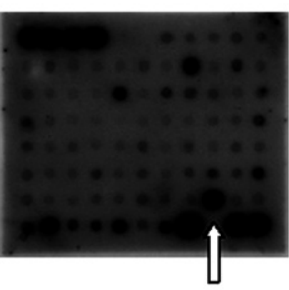

$\mathrm{K} 562+\mathrm{HS}-5$

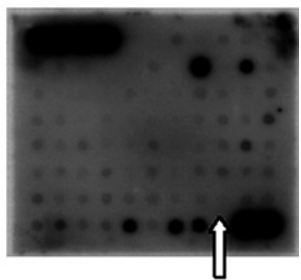

$\mathrm{K} 562+\mathrm{HS}-5$

Dox $0.2 \mu \mathrm{M}$

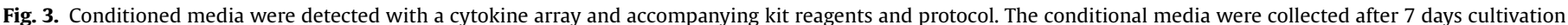

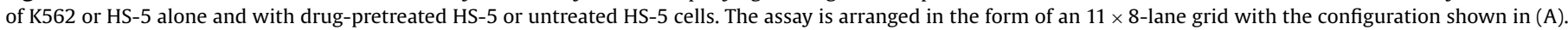

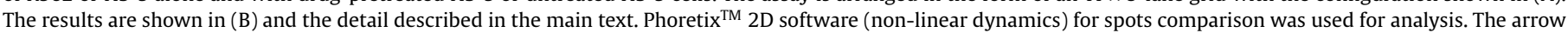
indicates the MIP- $3 \alpha$ spot in array membrane.

suggested that the increase in proliferation of co-cultured $\mathrm{K} 562$ cells resulted from the effect of anticancer drugs on HS-5 cells.

To address the CM effect on K562 cell growth, we tried to coculture K562 cells with CM from Ara-C-pretreated HS-5 cells. The data are shown in Fig. 2B. In this experiment, we cultured K562 cells only in CM without stromal cells, and it may slow down the effect on K562 cells. Obviously, the K562 cell number decreased (to $50 \%$ in CM from untreated HS-5 cells, and maintained proliferation (near to $88 \%$ ) in CM from $1.0 \mu \mathrm{M}$ Ara-C-pretreated HS-5 cells, in comparison to K562 cultured in normal medium. This indicated that some factors changed in HS- 5 cells after drug treatment.

Using phosphorylated $\mathrm{Rb}$ and Erk proteins as K562 cell proliferation markers (Razidlo et al., 2004; Alevizopoulos et al., 1997); we generated the Western blotting data shown in Fig. 2C. After 7 days cultivation, we measured the phosphorylation pattern of K562 cells after each treatment. In Fig. 2C (upper panel, lane 1), the results show a significant increase in the phosphorylation of $\mathrm{Rb}$ and Erk proteins, which indicated proliferation of $\mathrm{K} 562$ cells cultivated alone. Compared to K562 cells cultured alone, inhibition of proliferation of K562 cells co-cultured with HS-5 cells was due to cell differentiation, and the data showed a marked decrease in $\mathrm{Rb}$ and Erk phosphorylation (Fig. 2C, upper panel, lane 2). Additionally, K562 co-cultured with drug-pretreated HS-5 cells showed less Rb and Erk phosphorylation compared to K562 cells cultured alone. The data suggested that the change in microenvironment in $\mathrm{CM}$ triggered K562 cell proliferation (Fig. 2C, upper panel, lanes 3-6). The estimation of change in phosphorylation of $\mathrm{Rb}$ and Erk was based on the relative ratio to the control (K562 cells co-cultured with HS-5 cells) using a non-linear dynamic analysis method with the Phoretix 2D software. The data are shown in Fig. 2C, lower panel. The intensity of phosphorylation in both proteins revealed an increase in $\mathrm{K} 562$ cell proliferation after co-cultivation with drugpretreated HS-5 cells, and suggested recovery of cell proliferation.

\subsection{Cytokine array analysis}

We also utilized a membrane-bound antibody array technique to characterize the distribution of a wide range of bioactive trace 

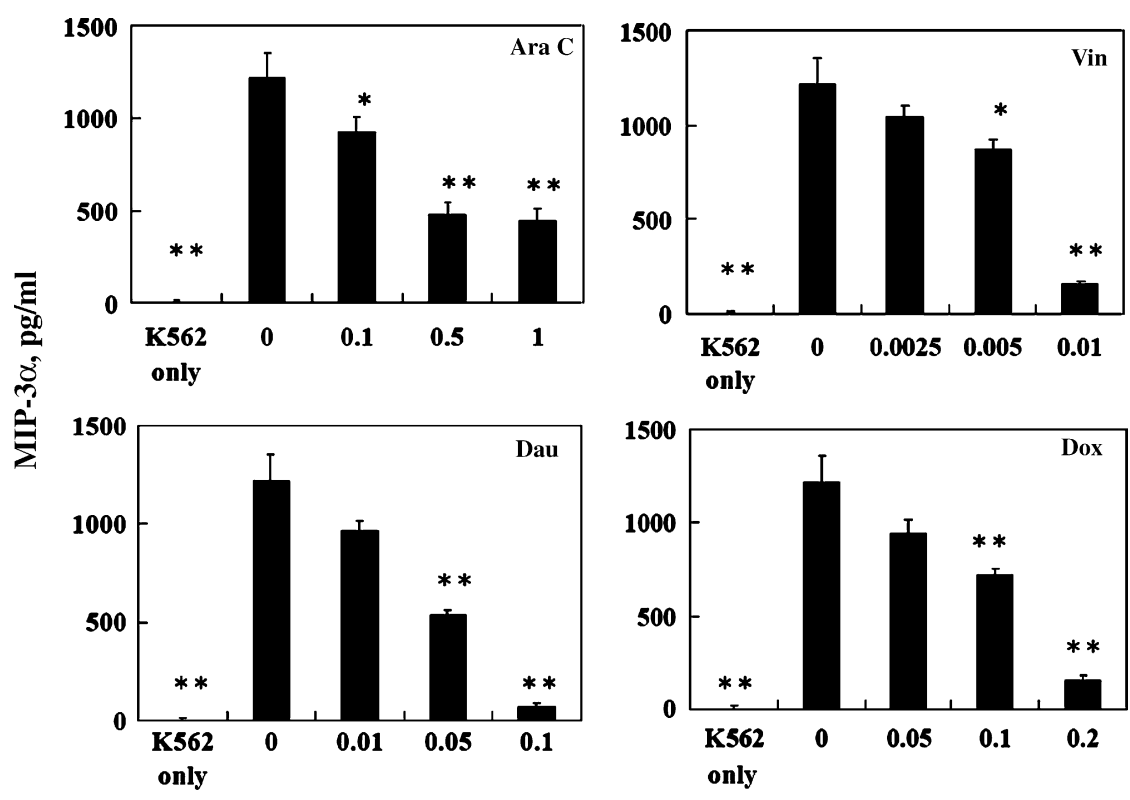

Concentration, $\mu \mathrm{M}$

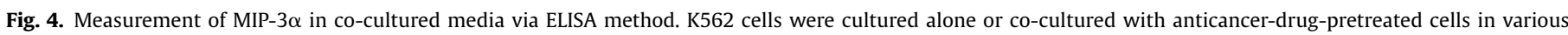

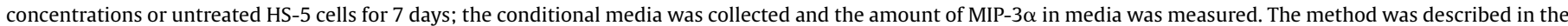
text. Results are presented as the mean \pm S.D. $(n \geq 8) .{ }^{*} p<0.05,{ }^{* *} p<0.01$. Significantly different from corresponding control group is shown at $p$-value.

proteins in the different CMs, which contained compounds secreted from stromal cells. In the array membrane, there were six identical positive control antibodies that contained a biotinylated protein standard (A1, B1, C1, D1, J8 and K8 in Fig. 3A) and three negative controls (E1, F1 and I8 in Fig. 3A). Fig. 3B depicts the results of a typical assay of a 1-ml CM sample using the array kit. Under these assay conditions, a reduced signal was detectable in cell-free culture medium that contained 5\% NCS (Fig. 3B); an intense signal was only observed for HGF, with far less intense signals present for the other cytokines. No significant signals appeared in the K562 culture medium (Fig. 3B), which suggests that the membrane array kit was far too insensitive to be used for the K562 cells cultured alone. We detected three intense signals in the media from HS-5 stromal cells cultured alone and co-cultured with K562 cells, including MIP-3 $\alpha$, IL-6 and TIMP-2 (Fig. 3B). The change in cytokines in CM should result from an intercellular reaction between HS-5 stromal cells and K562 cells. When K562 cells were co-cultured with drug-pretreated HS-5 cells, the secreted molecules in the CM were changed in decreasing way (Fig. 3B). Estimation of the change in these cytokines was based on the relative ratio to the control, using the non-linear dynamic analysis method with Phoretix 2D software. In the CM of drug-pretreated HS-5 cells co-cultivated with K562 cells, the level of MIP-3 $\alpha$ was significantly reduced. According to Fig. 1, the changes in cytokines in the CM may have resulted from the decrease in HS-5 cell viability. Although the data obtained were non-quantitative, at times we used them to obtain a rough estimate of the changes in some detectable cytokines.

\subsection{MIP-3 $\alpha$ with cell proliferation}

Based on the data in Fig. 3, we attempted to characterize the real changes in MIP- $3 \alpha$ level in CM. Using anti-MIP- $3 \alpha$ antibody; we measured MIP-3 $\alpha$ in K562 cells cultured alone or in drugpretreated co-cultured CM, using ELISA. Clearly, MIP-3 $\alpha$ levels decreased in drug-treated HS-5 cell medium in a dose-dependent manner (Fig. 4). The amount of MIP-3 $\alpha$ in CM of Ara-C-, vincristine, daunorubicin-, or doxorubicin-pretreated HS-5 cells decreased to $30,12.7,5.8$ and $12.7 \%$, respectively, at higher concentrations, com- pared to that from untreated HS-5 cells. The results imply that MIP-3 $\alpha$ plays an important role in $\mathrm{K} 562$ cell growth. They also indicated that the decrease in MIP- $3 \alpha$ levels in CM resulted from HS-5 cell damage induced by the drugs.

The decrease in MIP- $3 \alpha$ levels in CM provided a suitable microenvironment for K562 cell proliferation. To confirm whether MIP-3 $\alpha$ is an important factor for inhibiting K562 cell proliferation, we used MIP-3 $\alpha$-supplemented medium to analyze the $\mathrm{Rb}$ and Erk phosphorylation pattern. Clearly, the Rb and Erk protein phosphorylation decreased after 3 days co-cultivation with HS-5 cells, and in $1 \mathrm{ng} / \mathrm{ml}$ MIP-3 $\alpha$-supplemented medium (Fig. 5). The results with MIP-3 $\alpha$-supplemented cultivation indicated that initiation of $\mathrm{K} 562$ cell proliferation did result from the presence of MIP-3 $\alpha$ in the medium, and not from stromal cell death.

In our study, the doubling time of $\mathrm{K} 562$ cells was $24 \mathrm{~h}$ (data not shown). During this doubling process, the modification of proliferative molecules should progress; therefore, we chose 3 days cultivation for our phosphorylation assay. Upon adding various concentrations of MIP-3 $\alpha$ antibody to K562 cells cultured alone with MIP-3 $\alpha$, or co-cultured with HS- 5 cells for 3 days, the phosphoryla-

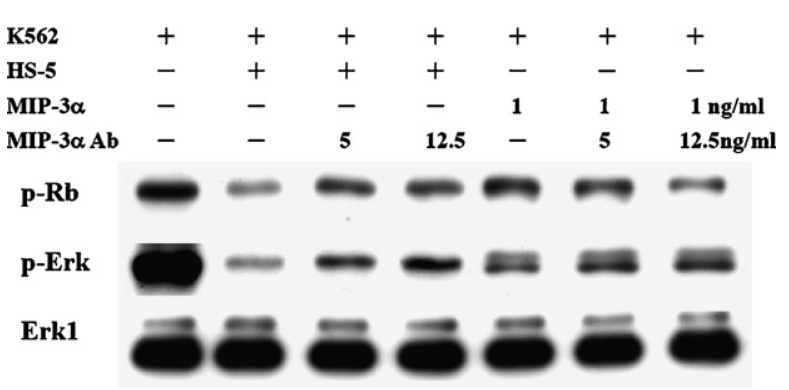

Fig. 5. Effect of MIP-3 $\alpha$ on phosphorylation of Rb and Erk proteins. K562 cells were cultured alone or co-cultured with HS- 5 in the absence or presence of $1.0 \mathrm{ng} / \mathrm{ml} \mathrm{MIP-}$ $3 \alpha$ or various amount of MIP- $3 \alpha$ antibody for 3 days then the cell protein extracts were harvested. The phosphorylation of $\mathrm{Rb}$ and Erk indicated the proliferation of $\mathrm{K} 562$ cells. K562 cell cultivation alone was used as a control. The anti-Erk antibody for recognizing Erk was used as an internal control. 
tion of $\mathrm{Rb}$ and Erk proteins was restored to $20-30 \%$. Such a level of antibody supplement may not be sufficient to completely neutralize the effect of MIP-3 $\alpha$ on K562 cell proliferation. Or, other minor factors in this microenvironment have synergistic effects on K562 cells. These data indicate that MIP- $3 \alpha$ may not be a unique factor, but it is important for inhibition of K562 cell proliferation.

\section{Discussion}

Little attention has been paid to the bone marrow stromal damage that results from anticancer drugs during chemotherapy (Schwartz et al., 1998). Previous studies have shown that postchemotherapy marrow stromal cells are deficient in supporting in vitro hematopoiesis, and have suggested that negative regulators induced by chemotherapy and cytokines may be the cause of this defect. In this study, we investigated the effects of stromal cells on growth of myeloid leukemia cell line K562. As a model for hematopoietic/stromal cell interaction, we used the stromal cell line HS-5, which has been shown to be capable of supporting hematopoiesis, and has recently been used for in vitro support of human progenitors (Torok-Storb et al., 1999; Goerner et al., 2000). Although our result did not confirm that HS-5 cells support myeloid cell differentiation, we found that K562 myeloid leukemia cells grow rapidly in co-cultivation with HS-5 cells pretreated with anticancer drugs. In general, increased differentiation of these cells leads to a halt in cell proliferation and vice versa (Steinman, 2002). This means that ongoing differentiation prevents proliferation. Our results indicated that the changes in diffusible molecules derived from HS-5 cells affected the proliferation of leukemic cells. We have shown above that four anticancer drugs reduced the activity of the HS-5 cell line and altered cytokine secretion. It may be assumed that cytokines secreted from stromal cells can cause defects in hematopoiesis or even prevent the differentiation of myeloid leukemic cells.

The bone marrow microenvironment is characterized by complex interaction between stromal cells, hematopoietic cells and cytokines. Bone marrow stromal cells have been shown to influence differentiation, growth and survival of normal hematopoietic cells through the secretion of a variety of cytokines (Bertrand et al., 2000). Using the cytokines array to analyze cytokine secretions from the HS-5 cell line, we identified cytokines that are potential targets of anticancer drugs. These cytokines might be functionally linked to their roles in K562 cell proliferation. Human hematopoietic cells can secrete numerous regulatory molecules that form the basis of intercellular cross-talk, networks and cell regulation in an autocrine and paracrine manner (Majka et al., 2001); stromal cells might be involved in intercellular cross-talk. Recent studies in mice have indicated that anticancer drugs, particularly at high doses, induce bone marrow stromal damage and/or dysfunction (Ben-Ishay and Barak, 2001). It has been shown that anticancer drugs affect cytokine secretion from stromal cells in vivo and have a suppression effect on hematopoiesis. In fact, our data showed that a large part of the secreted cytokines was decreased in $\mathrm{CM}$ from high-dose pretreated HS-5 cells. This suggests that anticancer drugs affect stromal cell activity by reducing cytokine secretion, or gene expression of HS-5 cells. The cytokines should have synergistic effect on cell differentiation and proliferation. It must be a complicated intercellular network, and further studies are needed to clarify this.

Chemokines are a large family of chemotactic cytokines that provide key signals for cell growth. The effect of MIP-3 $\alpha$ on cell proliferation and differentiation depends on cell type (Lisignoli et al., 2007; Hausmann et al., 2005). HS-5 cells can secrete MIP-3 $\alpha$ alone or in co-cultivation with K562 cells. After anticancer drug treatment, levels of MIP-3 $\alpha$ in media were found to decrease to a low level, and K562 cell proliferation increased significantly in medium from co-cultured cells. Inhibition of K562 cell proliferation in MIP-3 $\alpha$-supplemented medium indicates that MIP-3 $\alpha$ plays an important role in $\mathrm{K} 562$ cell growth. It also implies that the increase in K562 cell proliferation results from an absence of MIP- $3 \alpha$ in the medium from co-cultured cells. Our data suggest that MIP-3 $\alpha$ is involved in inhibition of K562 cell proliferation.

In summary, we showed that anticancer-drug-pretreated stromal cells have lost their ability to suppress proliferation of $\mathrm{K} 562$ cells, via a reduction in MIP- $3 \alpha$ or change in microenvironment. The mechanism of the MIP-3 $\alpha$ effect on K562 cells needs further study. However, the results imply that leukemia patients undergoing chemotherapy have deficient stromal cells, and a cytokine-deficient host microenvironment should be taken into account in cases of chemotherapeutic failure.

\section{Acknowledgements}

This work was supported by the National Science Council (NSC95-2314-B075-087) and Veterans General Hospital (V95S5004), Taiwan, ROC.

\section{References}

Alevizopoulos, K., Vlach, J., Honnecks, S., Amati, B., 1997. Cyclin E and c-Myc promote cell proliferation in the presence of pl6 $6^{\mathrm{INK} 4 \mathrm{a}}$ and of hypophosphorylated retinoblastoma family proteins. EMBO J. 16, 5322-5333.

Aoyagi, A., Aoyagi, M., Waga, K., Enokihara, H., 1996. Preferential inhibitory effect of soluble factor(s) in human bone marrow stromal cells on proliferation of K562 leukemia cells versus normal myeloid progenitor cells. Int. J. Hematol. 63, 205-213.

Badillo, A.T., Flake, A.W., 2006. The regulatory role of stromal microenvironments in fetal hematopoietic ontogeny. Stem Cell Res. 2, 241-246.

Barak, V., Ben-Ishay, Z., 1994. Cytokine and growth factor gene expression by bone marrow stroma of mice with damaged hematopoiesis and during regeneration. Leukemia Res. 18, 733-739.

Ben-Ishay, Z., Barak, V., 2001. Bone marrow stromal dysfunction in mice administered cytosine arabinoside. Eur. J. Haematol. 66, 230-237.

Bertrand, F.E., Eckfeldt, C.E., Fink, J.R., Lysholm, A.S., Pribyl, J.A., Shah, N., LeBien, W.T., 2000. Microenvironmental influences on human B-cell development. Immunol. Rev. 175, 175-186.

Cordero, E.A., Da Rocha Silla, L.M., Canedo, A.D., Allebrandt, W.F., Fogliatto, L., Nardi, N.B., 2004. Interaction between normal and CML hematopoietic progenitors and stroma influences abnormal hematopoietic development. Stem Cells Dev. 13, 225-228.

Frikin, F.C., Birner, R., Farag, S., 1993. Differential action of diffusible molecules in long-term marrow culture on proliferation of leukemic and normal haemotopoietic cells. Br. J. Haematol. 84, 8-15.

Garrido, S.M., Appelbaum, F.R., Willman, C.L., Banker, D.E., 2001. Acute myeloid leukemia cells are protected from spontaneous and drug-induced apoptosis by direct contact with a human bone marrow stromal cell line (HS-5). Exp. Hematol. $29,448-457$.

Goerner, M., Roecklein, B., Torok-Storb, B., Heimfeld, S., Kiem, H.P., 2000. Expansion and transduction of nonenriched human cord blood cells using HS-5 conditioned medium and FLT3-L. J. Hematother. Stem Cell Res. 9, 759-765.

Hausmann, M., Bataille, F., Spoettl, T., Schreiter, K., Falk, W., Schoelmerich, J., Herfarth, H., Rogler, G., 2005. Physiological role of macrophage inflammatory protein$3 \alpha$ induction during maturation of intestinal macrophages. J. Immunol. 175, 1389-1398.

Holstein, S.A., Hohl, R.J., 2001. Interaction of cytosine arabinoside and lovastatin in human leukemia cells. Leukemia Res. 25, 651-660.

Jiang, H., Sugimoto, K., Sawada, H., Takashita, E., Tohma, M., Gonda, H., 1998. Mutual education between hematopoietic cells and bone marrow stromal cells through direct cell-to-cell contact: factors that determine the growth of bone marrow stroma-dependent leukemic (HB-1) cells. Blood 92, 834-841.

Lisignoli, G., Piacentini, A., Cristino, S., Grassi, F., Cavallo, C., Cattini, L., Tonnarelli, B., Manferdini, C., Facchini, A., 2007. CCL20 chemokine induces both osteoblast proliferation and osteoclast differentiation: increased levels of CCL20 are expressed in subchondral bone tissue of rheumatoid arthritis patients. J. Cell Physiol. 210, 798-806.

Majka, M., Janowska-Wieczorek, A., Ratajczak, J., Ehrenman, K., Pietrzkowski, Z., kowalska, M.A., Gewirtz, A.M., Emerson, S.G., Ratajczak, M.Z., 2001. Numerous growth factors, cytokines and chemokines are secreted by human CD34(+) cells; myeloblast, erythroblasts and megakaryoblasts and regulate normal hematopoiesis in an autocrine/paracrine manner. Blood 97, 3075-3085.

Razidlo, G.L., Kortum, R.L., Haferbier, J.L., Lewis, R.E., 2004. Phosphorylation regulates KSR1 stability, ERK activation, and cell proliferation. J. Biol. Chem. 279, 47808-47814. 
Roecklein, B.A., Torok-Storb, B., 1995. Functionally distinct human marrow stromal cell lines immortalized by transduction with the papilloma virus E6/E7 genes. Blood 85, 997-1005.

Schwartz, G.N., Warren, M.K., Rothwell, S.W., Zujewski, J., Halverson, D.C., Cowan, K.H., Tolcher, A., O'Shanghnessy, J., Gress, R.E., 1998. Post-chemotherapy and cytokine pretreated marrow stromal cell layers suppress hematopoiesis from normal donor CD34 + cells. Bone Marrow Transplant. 22, 457-468.

Seymour, J.F., Huang, P., Plunkett, W., Gandhi, V., 1996. Influence of fludarabine on pharmacokinetics and pharmacodynamics of cytarabine: implications for a continuous infusion schedule. Clin. Can. Res. 2, 653-658.
Simmons, P.J., Przpiorka, D., Thomas, E.D., Torok-Storb, B., 1987. Host origin of marrow stromal cells following allogeneic bone marrow transplantation. Nature 328 , 429-432.

Steinman, R.A., 2002. Cell cycle regulators and hematopoiesis. Oncogene 21, 3403-3413.

Torok-Storb, B., Iwata, M., Graf, L., Gianotti, J., Horton, H., Byrne, M.C., 1999. Dissecting the marrow microenvironment. Ann. N.Y. Acad. Sci. 872, 164-170.

Wu, S., Korte, A., Kebelmann-Betzing, C., Gessner, R., Henze, G., Seeger, K., 2005 Interaction of bone marrow stromal cells with lymphoblasts and effects of prednisolone on cytokine expression. Leukemia Res. 29, 63-72. 